\title{
Piecewise rational approximation of square-root parameterizable curves using the Weierstrass form
}

\author{
Michal Bizzarri*,b, Miroslav Lávičkaa ${ }^{a, b}$, Jan Vršek ${ }^{a, b}$ \\ ${ }^{a}$ Department of Mathematics, Faculty of Applied Sciences, University of West Bohemia, \\ Univerzitni 8, 30614 Plzeñ, Czech Republic \\ ${ }^{b}$ NTIS - New Technologies for the Information Society, Faculty of Applied Sciences, University of West Bohemia, \\ Univerzitni 8, 30614 Plzeñ, Czech Republic
}

\begin{abstract}
In this paper we study situations when non-rational parameterizations of planar or space curves as results of certain geometric operations or constructions are obtained, in general. We focus especially on such cases in which one can identify a rational mapping which is a double cover of a rational curve. Hence, we deal with rational, elliptic or hyperelliptic curves that are birational to plane curves in the Weierstrass form and thus they are square-root parameterizable. We design a simple algorithm for computing an approximate (piecewise) rational parametrization using topological graphs of the Weierstrass curves. Predictable shapes reflecting a number of real roots of a univariate polynomial and a possibility to approximate easily the branches separately play a crucial role in the approximation algorithm. Our goal is not to give a comprehensive list of all such operations but to present at least selected interesting cases originated in geometric modelling and to show a unifying feature of the formulated method. We demonstrate our algorithm on a number of examples.
\end{abstract}

Key words: Square-root parameterization, hyperelliptic curves, Weierstrass form, topological graph, rational approximation

\section{Introduction}

Nowadays, the NURBS representation (where NURBS stands for №n- $\underline{\text { Uniform }} \underline{\text { Rational }} \underline{\mathrm{B}}$ - $\underline{\text { ppline}}$ ) is considered as a universal standard in geometric modelling and computer-aided design (CAD), see Piegl and Tiller (1997). This representation, based on parametric descriptions via polynomials and rational functions, offers a unifying exchanging data format that can be easily included into standard CAD systems. In particular, it enables to represent e.g. basic spline curves, conics, quadrics and many other elementary geometric objects originated in various technical applications.

However, most natural geometric operations applied to NURBS curves or surfaces do not preserve the rationality of the resulting objects. Among such operations belong offsetting, operation of convolution, construction of intersection curves of two surfaces, computing contours and isophotes on surfaces etc. Thus, investigation of the rationality still belongs to challenging and frequently studied problems in geometry and geometric modelling, see Hoschek and Lasser (1993); Farouki (2008). The first simple (generally) nonrational parametric descriptions are square-root parameterizations. A curve or surface $\mathcal{X}$ is called square-root parameterizable if it can be rationally parameterized in terms of $t$ or $u, v$ and $\sqrt{p(t)}$ or $\sqrt{p(u, v)}$, where $p(t)$ or $p(u, v)$ is a polynomial in $t$ or $u, v$, respectively. Then suitable approximate parameterization techniques must be used to overcome disadvantages of non-rational descriptions - see e.g. Sederberg et al. (1989);

\footnotetext{
* Corresponding author

Email addresses: bizzarri@ntis.zcu.cz (Michal Bizzarri), lavicka@kma.zcu.cz (Miroslav Lávička), vrsekjan@kma.zcu.cz (Jan Vršek)

Preprint submitted to Computer Aided Geometric Design
}

June 2, 2017 
Jüttler and Chalmovianský (2007); Pérez-Díaz et al. (2010); Rueda et al. (2013); Bizzarri and Lávička (2013b); Cheng et al. (2013) etc. We recall that the square-root parameterizations are typical for such operations when the number of points on the resulting curve/surface corresponding to the same input point on the given curve/surface equals two.

The main advantage of general approximation methods (but also of exact parameterization methods) is that they are universal. However, when a curve or a surface from a certain special class is given then it is suitable to exploit its distinguishing properties and formulate some modified (and simpler) method reflecting these special features. As concerns square-root parameterizable (i.e., rational, elliptic or hyperelliptic) curves, the birationality with planar Weierstrass curves is this extra feature which brings benefits. The computation of the topological graph of the Weierstrass curve is considerably simpler than for the general case, cf. Alcázar and Sendra (2005). Replacing the edges of the topological graph of the Weierstrass curve, which is easily constructible because of the nature of the Weierstrass equation, we compute a (piecewise) polynomial freeform curve and thus we find a (piecewise) rational approximation of the non-rational result of the studied geometric construction/operation.

\section{Motivation and preliminary}

Let a rational curve $\mathcal{C} \subset \mathbb{R}^{n}$ (where $n$ is typically 2 or 3 ) be given by a parameterization $\mathbf{x}(t)$. By Lüroth theorem, $\mathbf{x}(t)$ can be computed to be birational, i.e., generically it is a one-to-one rational map $\mathbf{x}: \mathbb{R} \rightarrow \mathcal{C}$. Hence its inverse provides a one-to-one map $\mathcal{C} \rightarrow \mathbb{R}$. From this point of view, the simplest class of non-rational curves are those possessing a two-to-one map $\mathcal{C} \rightarrow \mathbb{R}$.

Definition 2.1. A curve $\mathcal{C}$ is called hyperelliptic if and only if there exists a two-to-one map $\mathcal{C} \rightarrow \mathbb{R}$.

Let us note that hyperelliptic curves are usually assumed to have their genus greater than one. The curves of genus 0 , or 1 always possess such a map and they are called rational or elliptic, respectively. For the sake of simplicity, in what follows we call hyperelliptic all curves (including rational and elliptic) which doubly cover a line. It is well known that there exists a hyperelliptic curve of an arbitrary high genus. However the codimension of the set of all hyperelliptic curves in the space of all curves of genus $g$ is $g-2$ and thus a generic curve of genus at least 3 is not hyperelliptic. The following proposition is a classical result from algebraic geometry, cf. Hartshorne (1977).

Proposition 2.2. Each hyperelliptic curve of genus $g$ is birationaly equivalent to a planar curve in the Weierstrass form

$$
y^{2}-p(x)=0,
$$

where $p(x)$ is a square-free polynomial of degree $2 g+1$ or $2 g+2$.

The computational aspects and efficient algorithms for finding the Weierstrass form (implemented in CAS Maple) can be found in van Hoeij $(1995,2002)$. Nevertheless, in many situations originated in geometric modelling the Weierstrass form can be obtained directly without any subsequent transformation. Next, recall that a curve is called square-root parameterizable if there exists a square-free polynomial $p(t)$ and $\mathbf{x}(t, \sqrt{p(t)})=\left(x_{1}(t, \sqrt{p(t)}), \ldots, x_{n}(t, \sqrt{p(t)})\right)$ such that $x_{i}$ are rational expressions in $t$ and $\sqrt{p(t)}$ and for almost all points $\mathbf{p}$ on the curve there exists a unique $t_{0}$ such that $\mathbf{p}=\mathbf{x}\left(t_{0}, \sqrt{p\left(t_{0}\right)}\right)$. Note that such a parameterization is not technically a map but the occurrence of the square-root turns it into a multivalued map.

Lemma 2.3. A curve is square-root parameterizable via $\mathbf{x}(t, \sqrt{p(t)})$ if and only if it is birational to a Weierstrass curve in the form $y^{2}-p(x)=0$.

Proof. If $\mathbf{x}(t, \sqrt{p(t)})$ is a square-root parameterization of the curve $\mathcal{C}$ then $\mathbf{x}(x, y)$ is a rational mapping which maps the curve $\mathcal{E}: y^{2}-p(x)=0$ onto $\mathcal{C}$. Moreover since the parameterization is assumed to be generically injective so is the resulting mapping and thus $\mathcal{C}$ is birational to a Weierstrass curve. 
Conversely let $\phi: \mathcal{E} \rightarrow \mathcal{C}$ be a birational map from the Weierstrass curve $y^{2}-p(x)=0$ to some curve $\mathcal{C}$. Then the square root parameterization $(t, \sqrt{p(t)})$ of $\mathcal{E}$ composed with $\phi$ gives a square-root parameterization of $\mathcal{C}$.

Thus, we can assume in what follows that a curve in the Weierstrass form is the input for further considerations. Since the mapping $\mathbf{x}(x, y)$ is in fact a rational map

$$
\mathbf{x}: \mathbb{R}^{2} \rightarrow \mathbb{R}^{n}
$$

which sends $\mathcal{E}$ to $\mathcal{C}$, then we will construct a collection of polynomial $\operatorname{arcs} \mathbf{f}_{i}(t)$ approximating the Weierstrass curve $\mathcal{E}$ and using

$$
t \mapsto \mathbf{x}\left(\mathbf{f}_{i}(t)\right), \quad i=1, \ldots, k
$$

we arrive at a piecewise rational curve replacing the original non-rational curve $\mathcal{C}$.

\section{Rational approximation of curves in the Weierstrass form}

For the later use, we start with short recalling some fundamental notions which are necessary for constructing the topological graphs of algebraic curves. A point $\mathbf{p}$ on a planar curve $\mathcal{C}$ defined by $f(x, y)=0$ is called a $x$ - or $y$-critical if $f_{y}(\mathbf{p})=0$ or $f_{x}(\mathbf{p})=0$, respectively. All these points are denoted by a unified name critical points. The points on $\mathcal{C}$ which are simultaneously $x$ - and $y$-critical are the singular points of $\mathcal{C}$. For more details see e.g. Alcázar and Sendra (2005); Bizzarri and Lávička (2013b). By the topological graph of a real algebraic curve we understand a construction of some arrangement of polylines which is topologically equivalent to the given curve, see Gamelin and Greene (1999).

Now, we introduce a method for computing an approximate (piecewise) rational parametrization of a hyperelliptic curve $\mathcal{E}$ in the Weierstrass form - preferably in some prescribed bounding box, otherwise it is specially emphasized. We proceed in two steps:

(i) first, we construct the topological graph of $\mathcal{E}$ having the critical points and the inflection points of $\mathcal{E}$ as its vertices;

(ii) next, we replace each edge of the graph by some free-form curve and optimize its shape.

As concerns PART (i), any general method for constructing topological graphs of planar curves can be employed, see for instance Hong (1996); Cheng et al. (2009); Bizzarri and Lávička (2013b). However when a planar curve is given in the Weierstrass form (1) the construction of the topological graph is considerably simpler than for the general case. Since we work with a function in one variable in this case, it is easy to compute the critical and inflection points (for instance, no elimination technique is needed). Moreover it is not necessary to determine the number of branches starting at the critical points, cf. Bizzarri and Lávička (2013b), and therefore it is straightforward to join the corresponding pairs. In addition, we do not have to deal with the singular points.

In PART (ii) we replace all edges of the graph by some (parts of) free-form curves, which will approximate the original curve $\mathcal{E}$, we prescribe all the critical and inflection points of $\mathcal{E}$ to the set of vertices of the topological graph; this improves the consequent computation of the approximant. Such a graph is usually called a graph of critical points of the curve $\mathcal{E}$ and denoted by $G(\mathcal{E})$. Let us emphasize that it brings another simplification compared to general methods, cf. Hong (1996); Cheng et al. (2009); Bizzarri and Lávička (2013b), as no other extra points are needed for constructing the topological graphs. Furthermore, we can exploit a useful fact that the curve $\mathcal{E}$ is composed of two symmetrical parts $\mathcal{E}^{ \pm}$, i.e., graphs of the functions $y= \pm \sqrt{p(x)}$. Hence it is enough to deal only with e.g. $\mathcal{E}^{+}$given by the function $y=\sqrt{p(x)}$ and use the symmetry to obtain the results for $\mathcal{E}^{-}$.

Let us now present the method in more detail. We start with computing the real roots (lying in some prescribed interval of interest $\left.\left[a_{0}, a_{1}\right]\right)$ of the polynomials in one variable $p(x), p^{\prime}(x)$ and $2 p^{\prime \prime}(x) p(x)-$ $p^{\prime}(x)^{2}$ which correspond to the $x$-critical, $y$-critical and inflection points (of a corresponding part) of $\mathcal{E}^{+}$, 
respectively. More precisely, if $x_{i}$ is a real root of the polynomial $p(x), p^{\prime}(x)$ or $2 p^{\prime \prime}(x) p(x)-p^{\prime}(x)^{2}$, then the point $\left(x_{i}, \sqrt{p\left(x_{i}\right)}\right)$ is a real $x$-critical (i.e., $\left(x_{i}, 0\right)$ in this case), $y$-critical or inflection point on $\mathcal{E}^{+}$, respectively. Thus when sorting all the roots (lying in $\left[a_{0}, a_{1}\right]$ together with the boundary values $x_{0}=a_{0}, a_{1}=x_{k+1}$ ) and computing the corresponding real points $\left(x_{i}, \sqrt{p\left(x_{i}\right)}\right)$ (the points satisfying $\left.p\left(x_{i}\right) \geq 0\right)$ it is enough to connect the consecutive pairs $\left(x_{i}, \sqrt{p\left(x_{i}\right)}\right)$ and $\left(x_{i+1}, \sqrt{p\left(x_{i+1}\right)}\right)$ assuming the polynomial $p(x)$ is non-negative on $\left[x_{i}, x_{i+1}\right]$.

Now we can start with PART (ii) and replace each edge of the topological graph $G(\mathcal{E})$ representing any real segment $\mathcal{E}_{i}$ of $\mathcal{E}$ by a suitable $\operatorname{arc} \mathbf{f}_{i}(t)$ of the Ferguson cubic (or any other free form curve when needed), cf. Farin et al. (2002), which possesses the same tangent lines at its endpoints (vertices of $G(\mathcal{E})$ ) as $\mathcal{E}$ has. The tangent vectors at the points $\mathbf{p}_{i}=\left(x_{i}, y_{i}\right)$ are equal to

$$
\mathbf{t}_{i}=\left(2 \sqrt{p\left(x_{i}\right)}, p^{\prime}\left(x_{i}\right)\right) .
$$

Especially, these are the multiples of the unit vectors $(0,1)$ and $(1,0)$ for $x$-and $y$-critical points, respectively.

Thus for each edge of $G\left(\mathcal{E}^{+}\right)$connecting the points $\mathbf{p}_{i}$ and $\mathbf{p}_{i+1}$ we construct the Ferguson cubic $\mathbf{f}_{i}\left(t, \alpha_{i}, \beta_{i}\right)$ interpolating these two points and the corresponding tangent vectors $\mathbf{t}_{i}$ and $\mathbf{t}_{i+1}$ with the lengths $\alpha_{i}, \beta_{i}$ which serve as free modelling shape parameters. Next, the particular values $\widehat{\alpha}_{i}, \widehat{\beta}_{i}$ of the lengths $\alpha_{i}, \beta_{i}$ are computed w.r.t. some natural criteria. For instance we can require that the curvatures of the Ferguson cubics $\mathbf{f}_{i}\left(t, \alpha_{i}, \beta_{i}\right)$ at $\mathbf{p}_{i}$ and $\mathbf{p}_{i+1}$ are equal to the curvatures of $(x, \sqrt{p(x)})$ at these points.

Of course any other criterion or approximation method can be used. For instance one can always compute $\widehat{\alpha}_{i}, \widehat{\beta}_{i}$ by minimizing the objective function

$$
\Phi_{i}\left(\alpha_{i}, \beta_{i}\right)=\int_{0}^{1} \frac{f^{2}\left(\mathbf{f}_{i}\left(t, \alpha_{i}, \beta_{i}\right)\right)}{\left\|\nabla f\left(\mathbf{f}_{i}\left(t, \alpha_{i}, \beta_{i}\right)\right)\right\|^{2}} \mathrm{~d} t,
$$

which measures the orthogonal distance of the parametric curve $\mathbf{f}_{i}\left(t, \alpha_{i}, \beta_{i}\right)$ to the implicitly given curve $f(x, y)=y^{2}-p(x)=0$, see Bizzarri and Lávička (2013b) for further details.

Finally, using the symmetry we obtain a (piecewise) polynomial approximation of the whole curve $\mathcal{E}$.

Example 3.1. Consider (a part of) the cubic $\mathcal{E}$ of genus 1 in the form

$$
y^{2}-x(x-1)(x-2)=0, \quad x \in[0,3] .
$$

We start with PART (i) and construct the graph of critical points of $\mathcal{E}^{+}$. The polynomial $p(x)=x(x-1)(x-2)$ has three real roots $0,1,2$, which together with the boundary points of the interval $[0,3]$ form a sequence of points $\{0,1,2,3\}$. We divide the sequence into the subsequences such that $p(x)$ is non-negative on the whole intervals given by the boundary values of these subsequences, i.e., we arrive at

$$
I_{1}=\{0,1\}, \quad I_{2}=\{2,3\} .
$$

Next the polynomial $p^{\prime}(x)$ has two real roots $1 \pm 1 / \sqrt{3}$, however only $1-1 / \sqrt{3}$ gives a non-negative value of $p(x)$. Hence inserting this value to (7) yields two new sequences

$$
\bar{I}_{1}=\left\{0,1-\frac{1}{\sqrt{3}}, 1\right\}, \quad \bar{I}_{2}=\{2,3\} .
$$

The polynomial $2 p^{\prime \prime}(x) p(x)-p^{\prime}(x)^{2}$ has two real root equal to $1 \pm \sqrt{1+2 / \sqrt{3}}$. But only one of them lies in the considered interval and hence it will be added to the sequence, i.e., we have

$$
\tilde{I}_{1}=\left\{0,1-\frac{1}{\sqrt{3}}, 1\right\}, \quad \tilde{I}_{2}=\left\{2,1+\sqrt{1+\frac{2}{\sqrt{3}}}, 3\right\} .
$$



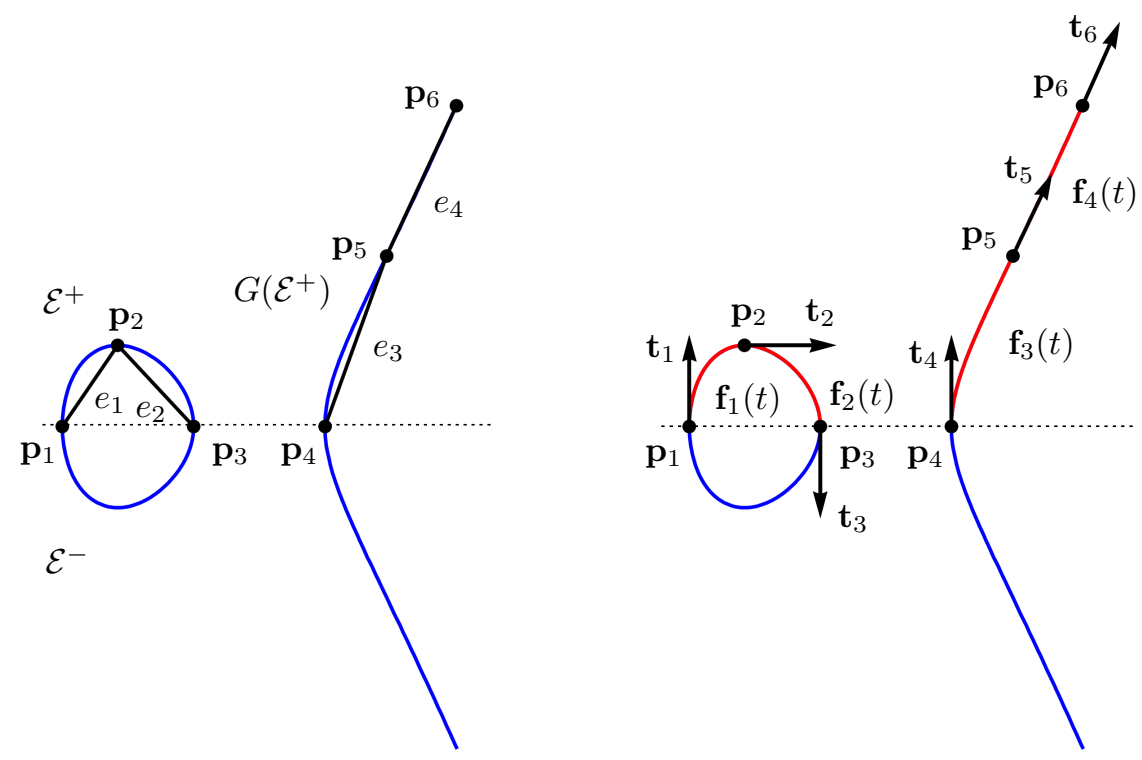

Figure 1: Left: The topological graph $G\left(\mathcal{E}^{+}\right)$(black) of the upper part $\mathcal{E}^{+}$of the hyperelliptic cubic curve $\mathcal{E}$ in the Weierstrasse form (blue) from Example 3.1. Right: Its polynomial approximation (red).

Now we compute the corresponding $y$-coordinates of $\mathcal{E}^{+}$yielding the vertices of the graph of critical points $G\left(\mathcal{E}^{+}\right)$, i.e.,

$$
P_{1}=\left\{(0,0),\left(1-\frac{1}{\sqrt{3}}, \frac{\sqrt{2}}{\sqrt[4]{81}}\right),(1,0)\right\}, \quad P_{2}=\left\{(2,0),\left(1+\sqrt{1+\frac{2}{\sqrt{3}}}, \sqrt{\frac{2}{3}} \sqrt[4]{3+2 \sqrt{3}}\right),(3, \sqrt{6})\right\} .
$$

The edges of $G\left(\mathcal{E}^{+}\right)$are the joints of the vertices in any individual sequence $P_{i}$, see Fig. 1 (left).

As concerns PART (ii), we compute the corresponding unit tangent vectors at the prescribed points and construct three Ferguson cubics interpolating the consecutive points from the sequences $P_{1}$ and $P_{2}$ having the corresponding tangent vectors with their lengths as free shape parameters. Finally computing the particular values $\widehat{\alpha}_{i}$ and $\widehat{\beta}_{i}$ such that $\mathbf{f}_{i}\left(t, \alpha_{i}, \beta_{i}\right)$ possess the same curvatures at $\mathbf{p}_{i}$ and $\mathbf{p}_{i+1}$ as the curve $\mathcal{E}^{+}$yields the piecewise approximate parametric description $\mathbf{f}_{i}^{+}(t), i=1,2,3,4$, of $\mathcal{E}^{+}$for $x \in[0,4]$, see Fig 1 (right). Of course the whole piecewise polynomial parameterization can be obtained by symmetry just considering also $\mathbf{f}_{i}^{-}(t)=\left(f_{i 1},-f_{i 2}\right)$.

In the previous part we introduced a simple method how the curve $\mathcal{E}$ in the Weierstrass form $y^{2}=p(x)$ can be approximated by Ferguson cubics within a prescribed bounding box given by some real interval $\left[a_{0}, a_{1}\right]$. However in various problems originated in geometric modelling it may be convenient to approximate $\mathcal{E}$ for $x \in(-\infty,+\infty)$. Hence in the rest of this section we discuss the situations when the interval of interest is the whole parameter domain $\mathbb{R}$.

In particular we must distinguish three possible cases (see Fig. 2) which represent typical situations w.r.t. the number of bounded and unbounded components of the curves in Weierstrass form: (i) the curve $\mathcal{E}$ is composed only of real closed parts (one or more); (ii) the curve $\mathcal{E}$ consists of several (at least two) parts whereas at least one of them is unbounded; (iii) $\mathcal{E}$ is composed of two (upper and lower ) symmetric parts which are real for all $x \in \mathbb{R}$. All of these types can be easily derived from the roots of $p(x)$.

As concerns Situation (i), i.e., the polynomial $p(x)$ contains at least two real roots, has even degree and its leading coefficient is negative. Then the designed approach can be used unchanged without considering 
the boundary values $a_{0}$ and $a_{1}$.

Situation (ii) means that $p(x)$ contains at least one real root and it is different from Situation (i). This type can be solved by providing the birational transformation

$$
x \mapsto \frac{1}{x}-m, \quad y \mapsto \frac{y}{x^{\lceil n / 2\rceil}},
$$

where $n$ is the degree of $p(x)$ and $m$ is a real number chosen such that $p(m)<0$. Then we obtain a bounded curve $\mathcal{E}^{\prime}$ in the Weierstrass form

$$
y^{2}=\sum_{i=0}^{n} \alpha_{i} x^{2\lceil n / 2\rceil-i}, \quad \alpha_{i} \in \mathbb{R} .
$$

Next, we approximate $\mathcal{E}^{\prime}$ and using the inverse transformation to (11), i.e.,

$$
x \mapsto \frac{1}{x+m}, \quad y \mapsto \frac{y}{(x+m)^{\lceil n / 2\rceil}},
$$

yields the approximation of $\mathcal{E}$, cf. Examples 4.6 and 4.7 .

Finally, in Situation (iii) the polynomial $p(x)$ has only complex roots and a positive leading coefficient. Then we start with approximating one real part of $\mathcal{E}$ for $x \in\left[a_{0}, a_{1}\right]$ as in the original approach. Next, the remaining parts of $\mathcal{E}$ are approximated separately by passing to the curves $\mathcal{E}^{\prime}$ using (11), parameterizing $\mathcal{E}^{\prime}$ for $x \in\left[1 / a_{0}, 1 / a_{1}\right]$ and going back using (13), see Examples 5.16 and 5.11.
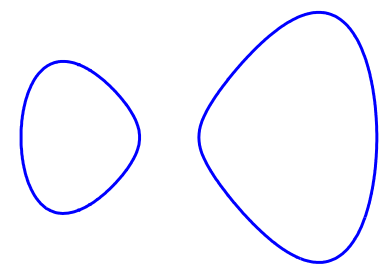

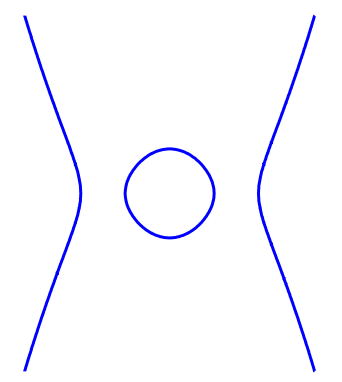

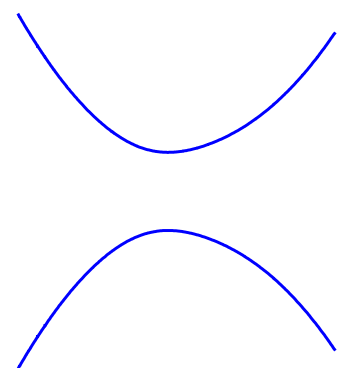

Figure 2: Three types of curves in the Weierstrass form with respect to the methods used for approximating their components - Left: Situation (i), Middle: Situation (ii), and Right: Situation (iii).

Remark 3.2. One of the advantages of the presented method is that it is simple to construct the approximation of the Weierstrass curve with the correct topology. Since the topological graph contains $x$-, $y$-critical and inflection points as its vertices it is possible to construct the C-shape approximants as the Bézier curves lying in the convex hull of their (mutually non-intersecting) control polygons. Let us emphasize that the $\mathrm{C}$-shape is guaranteed by a right choice of the lengths of the tangent vectors (i.e., the legs of the control polygons do not cross), see Fig. 3

Remark 3.3. One of the goals of the designed method is to use a number of approximating arcs as small as possible. As mentioned above there is no problem with the approximation of the Weierstrass curve. However, the problems can sometimes occur when applying mapping $\mathbf{x}=\left(\phi_{1} / \phi_{0}, \ldots, \phi_{n} / \phi_{0}\right)$, see (2). Hence in some situations it is necessary to have more vertices in the constructed graph to guarantee a right shape of the approximating curve.

From these reasons we add the real intersections of the Weierstrass curve $\mathcal{E}$ with the curve $\phi_{0}=0$ (if they exist). We recall, that these points are either mapped to infinity, or are the base-points of $\mathbf{x}$. 


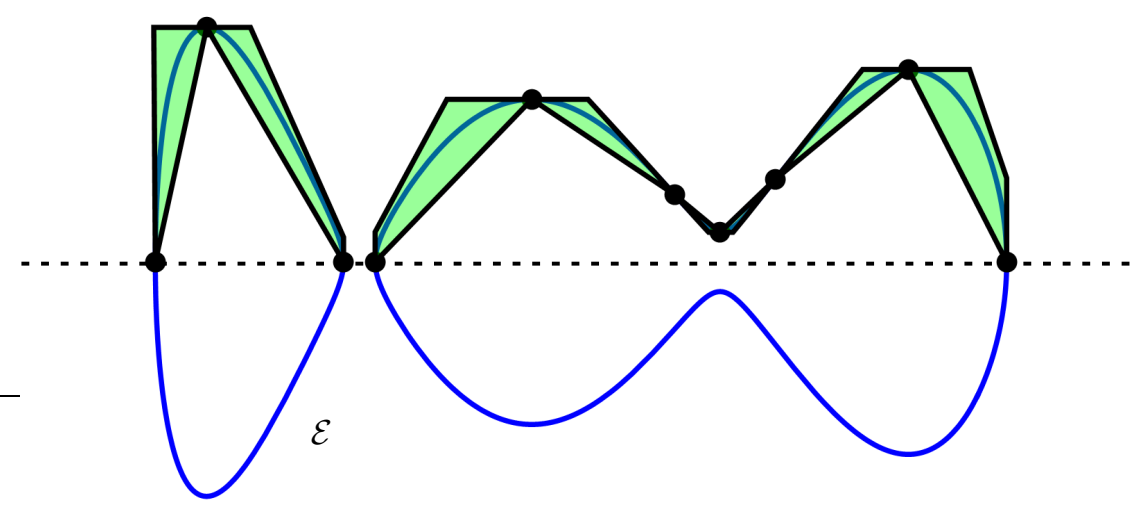

Figure 3: Bézier cubics with mutually non-intersecting control polygons (having legs which also do not cross) approximating the Weierstrass curve and guaranteeing its right topology, see Remark 3.2.

\section{Hyperelliptic curves on surfaces}

In this section we pay attention to distinguished curves on rational surfaces which do not have to be rational, in general. Especially we are interested in situations in which one can identify a rational mapping such that its fiber at a generic point consists of two points, in other words when this mapping is a double cover of a rational curve. Then we arrive at hyperelliptic curves, which are birational to plane curves in the Weierstrass form, and the designed approximate method can be immediately applied.

\subsection{Contour curves on canal surfaces}

A canal surface $\mathcal{S}$ is the envelope of a 1-parameter family of spheres $F$ whose centers trace a curve $\mathbf{m}(t)$ in $\mathbb{R}^{3}$ and possess radii $r(t)$, i.e.,

$$
F(t):\|\mathbf{x}-\mathbf{m}(t)\|^{2}-r(t)^{2}=0, \quad t \in I,
$$

where $\mathbf{x}=(x, y, z)$ and $\mathbf{m}^{\prime}(t)^{2}-r^{\prime}(t)^{2} \geq 0$ guaranteeing the real envelope. The curve $\mathbf{m}(t)$ is called the spine curve and $r(t)$ the radius function of $\mathcal{S}$. Next, each sphere $F(t)$ contributes to the canal surface by the so called characteristic circle $C(t)$, which is the intersection of the sphere $F(t)$ and the plane $\mathrm{d} F(t) / \mathrm{d} t$.

In Bizzarri and Lávička (2013a); Bizzarri et al. (2015, 2016) special curves on canal surfaces providing the whole family of rational canal surfaces sharing the same silhouette were investigated. These curves, along which the surface normals are parallel with a prescribed plane, are suitable in special cases for computing rational parameterizations of canal surfaces. However, they are not rational in general. We will deal with these curves in more detail.

Definition 4.1. A contour curve w.r.t. a vector $\mathbf{v}$ is the locus of all points at which the surface normal is perpendicular to the vector $\mathbf{v}$.

As derived in Bizzarri et al. (2015), for the canal surface with the rational spine curve $\mathbf{m}(t)$ and the rational radius function $r(t)$, the contour curve can be parameterized as

$$
\mathbf{c}_{\mathbf{v}}^{ \pm}=\mathbf{m}-r \frac{r^{\prime}\left(\mathbf{m}^{\prime}-\left(\mathbf{m}^{\prime} \cdot \mathbf{v}\right) \mathbf{v}\right) \pm\left(\mathbf{m}^{\prime} \times \mathbf{v}\right) \sqrt{\left\|\mathbf{m}^{\prime}\right\|^{2}-\left(\mathbf{m}^{\prime} \cdot \mathbf{v}\right)^{2}-r^{\prime 2}}}{\left\|\mathbf{m}^{\prime}\right\|^{2}-\left(\mathbf{m}^{\prime} \cdot \mathbf{v}\right)^{2}} .
$$

Let us rewrite

$$
\sqrt{\left\|\mathbf{m}^{\prime}\right\|^{2}-\left(\mathbf{m}^{\prime} \cdot \mathbf{v}\right)^{2}-r^{\prime 2}}=\frac{a(t)}{b(t)} \sqrt{p(t)},
$$

where $p(t)$ is a square-free polynomial. Using Lemma 2.3 we arrive at: 
Corollary 4.2. Any irreducible contour curve on a canal surface w.r.t. a direction $\mathbf{v}$ is a hyperelliptic curve with Weierstrass form (1), where polynomial $p$ is from (16).

We can approximate the Weierstrass curve $\mathcal{E}$ and provide substitution (3), see the following example.

Example 4.3. Consider a (part of) canal surface given by

$$
\mathbf{m}(t)=\left(t, t^{2}, t^{3}\right), \quad r(t)=\frac{1}{4}\left(t^{2}+1\right), \quad t \in[-1,1] .
$$

Then the parametric equation of the contour curve w.r.t. the vector

$$
\mathbf{v}=\left(\frac{1}{\sqrt{3}}, \frac{1}{\sqrt{3}}, \frac{1}{\sqrt{3}}\right)
$$

contains the square-root of the polynomial $p$, cf. (16),

$$
p(t)=72 t^{4}-48 t^{3}+5 t^{2}-16 t+8 .
$$

So we approximate the upper part of the curve $\mathcal{E}: y^{2}-p(t)=0$ and substitute it to (15) to obtain a rational approximation of the considered contour curve, see Fig. 4.
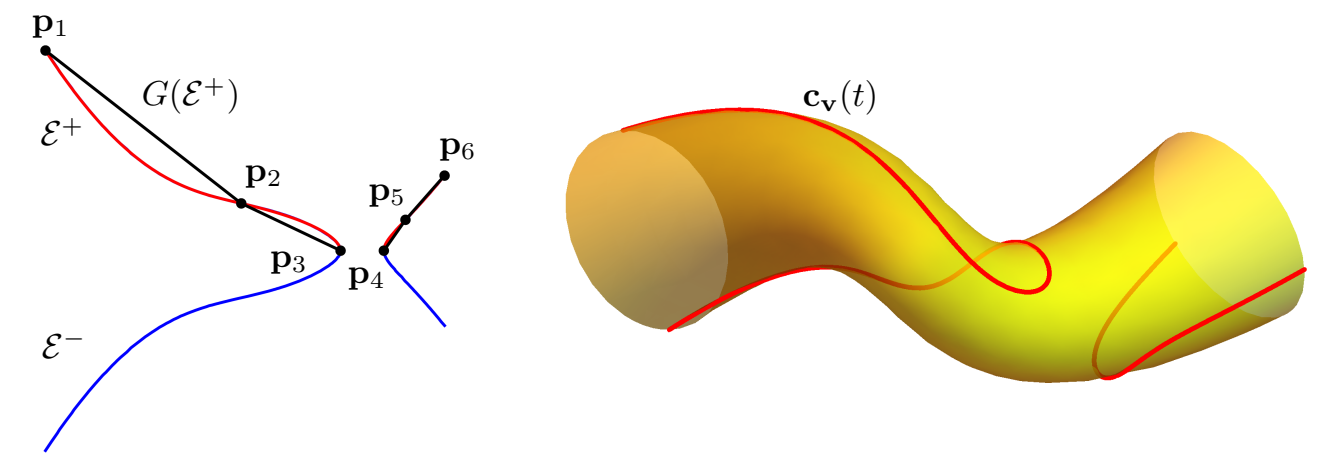

Figure 4: The graph of critical points $G\left(\mathcal{E}^{+}\right)$of a curve $\mathcal{E}$ (left) and the canal surface with the rational approximation of the contour curve (right) from Example 4.3.

\subsection{Intersection of ruled surfaces with quadrics}

The rational-ruled-surface-to-quadric intersection is a particular example of a general surface-to-surface intersection problem. Let us emphasize that the intersection curve of two surfaces is getting complicated very quickly when the degrees of the input surfaces grow. And moreover, it is almost never rational. The examples of surface-to-surface intersections producing hyperelliptic curves are for instance:

1. a rational surface with a cubic parameterization and a plane,

2. a surface containing a pencil of conics and a plane,

3. a rational ruled surface and a quadric.

Whereas the second class contains for example canal or ringed surfaces as a subclass, we will deal with the third case. The reason is that it possesses nice geometric consequences. For example, it enables us to approximate isophotes on ruled surfaces. And in the next section we will see that the offsets and some bisectors can be treated in this way as well. Moreover if we allow the field extension from $\mathbb{R}$ to $\mathbb{C}$ then we arrive at the algorithm for the quadric-quadric intersection for free, because all quadrics are ruled surfaces over the algebraically closed field $\mathbb{C}$. 
A rational ruled surface is a surface $\mathcal{R}$ parameterizable in the form

$$
\mathbf{x}(u, v)=\mathbf{a}(u)+v \mathbf{b}(u)
$$

where $\mathbf{a}(u)$ and $\mathbf{b}(u)$ are rational expressions. Therefore it is generated by a rational family of lines, called rulings. There exists a rational map $\phi: \mathcal{R} \rightarrow \mathbb{R}$ contracting the lines, i.e., $\phi^{-1}\left(u_{0}\right)=\left\{\mathbf{a}\left(u_{0}\right)+v \mathbf{b}\left(u_{0}\right) \mid v \in\right.$ $\mathbb{R}\}$.

Let $\mathcal{Q}$ be a quadric different from $\mathcal{R}$. Then $\mathcal{Q} \cap \mathcal{R}$ consists of finitely many rulings and some curve $\mathcal{C}$. For the sake of simplicity assume that $\mathcal{C} \not \subset \operatorname{Sing}(\mathcal{R})$. Since each ruling intersects $\mathcal{Q}$ twice (possibly at infinity and/or in complex extension) we may conclude that $\mathcal{C}$ intersects each ruling twice as well. Now there are two possibilities. First $\mathcal{C}$ may be reducible in which case all the components are rational and can be parameterized directly, or it is irreducible and the restriction $\left.\phi\right|_{\mathcal{C}}: \mathcal{C} \rightarrow \mathbb{R}$ is a double cover. Since the rational curves were assumed to belong among hyperelliptic curves we may conclude.

Corollary 4.4. Any irreducible component of the intersection of a rational ruled surface with a quadric is a hyperelliptic curve.

In what follows we will demonstrate the approximation of the intersection curve of a ruled surface with a quadric on two particular applications. We start with computing isophotes.

Definition 4.5. Let $\mathbf{v} \in \mathbb{R}^{3}$ be a unit vector and $\alpha \in(0, \pi / 2)$. Denote $\mathbf{n}_{\mathbf{p}}$ the normal vector at a smooth point $\mathbf{p}$ on the surface $\mathcal{R}$. Then the isophote $\mathcal{I}_{\mathbf{v}, \alpha}$ on $\mathcal{R}$ w.r.t. the direction $\mathbf{v}$ and angle $\alpha$ is the closure of the set

$$
\left\{\mathbf{p} \in \mathcal{R} \backslash \operatorname{Sing}(\mathcal{R}) \mid\left(\mathbf{n}_{\mathbf{p}} \cdot \mathbf{v}\right)^{2}=\cos \alpha\left(\mathbf{n}_{\mathbf{p}} \cdot \mathbf{n}_{\mathbf{p}}\right)\right\} .
$$

Hence the isophote consists of the points where the surface normals enclose the angle $\alpha$ or $-\alpha$ with the given direction. Let us stress that choosing the angle $\alpha=\pi / 2$ would lead to a special instance of the isophotes, i.e., to the so called contour curves (mentioned for canal surfaces in the previous subsection), see also Vršek (2016) for more details about contour curves and isophotes on ruled surfaces.

If the tangent plane at a regular point $\mathbf{p}$ possesses the equation $\mathbf{n}_{\mathbf{p}} \cdot \mathbf{x}+h_{\mathbf{p}}=0$ then this equation is unique up to a nonzero scalar multiple. We can assign to the regular point $\mathbf{p}$ a point with the coordinates $\left(\mathbf{n}_{\mathbf{p}}, h_{\mathbf{p}}\right)$ in the projective space $\mathbb{P}_{\mathbb{R}}^{3}$. If the ruled surface $\mathcal{R}$ is non-developable then this mapping is birational and the image is the dual surface $\mathcal{R}^{\vee}$. It is easy to show, see e.g. Pottmann and Wallner (2001), that $\mathcal{R}^{\vee}$ is again a ruled surface. Now the isophote is nothing but the preimage of the intersection of $\mathcal{R}^{\vee}$ with the quadric defined by (21) under this duality mapping. Thus it can be viewed as the intersection of a ruled surface with a quadric.

Nonetheless, in order to obtain the Weierstrass form it is not necessary to translate the problem to the dual language. If we start with parametric representation (20) of $\mathcal{R}$ then the normal field can be parameterized as

$$
\mathbf{n}(u, v)=\mathbf{n}_{1}(u)+v \mathbf{n}_{2}(u)
$$

where $\mathbf{n}_{i}(u)$ are polynomial curves in $\mathbb{R}^{3}$. Hence substituting this into (21) leads to the pull-back of the isophote into parameter plane. This is a curve defined by the equation $a(u) v^{2}+b(u) v+c(u)=0$, where $a, b, c \in \mathbb{R}[u]$ are polynomials without a common factor. The transformation $u=x, v=\frac{-b(x)+y}{2 a(x)}$ maps this curve into a curve in the Weierstrass form

$$
y^{2}=b^{2}(x)-4 a(x) c(x),
$$

which can be approximated by the techniques introduced in Section 3 .

Example 4.6. It was mentioned above that all quadrics are ruled surfaces over the algebraically closed field $\mathbb{C}$. So we will present an example with isophotes on a quadric. Consider the parametrically given ellipsoid

$$
\mathbf{x}(u, v)=\left(\frac{4 u}{u^{2}+v^{2}+1}, \frac{2 v}{u^{2}+v^{2}+1}, \frac{u^{2}+v^{2}-1}{u^{2}+v^{2}+1}\right), \quad u, v \in \mathbb{R}
$$


the unit vector $\mathbf{v}=(1 / \sqrt{2}, 0,1 / \sqrt{2})$ and the angle $\alpha=\pi / 3$. Then the isophote $\mathcal{I}_{\mathbf{v}, \alpha}$ corresponds to the curve $\mathcal{C}_{\mathbf{v}, \alpha}$ of genus 1 , see Fig. 5 (a)

$$
3 u^{4}+8 u^{3}+6 u^{2} v^{2}-3 u^{2}+8 u v^{2}-8 u+3 v^{4}-10 v^{2}+3=0 .
$$

We compute the corresponding curve $\mathcal{E}$ in the Weierstrass form, cf. van Hoeij (1995)

$$
y^{2}=-x^{3}+\frac{4048 x}{3}+\frac{48512}{27} .
$$

Let us emphasize that $\mathcal{E}$ is unbounded, see Fig. 5 (b), although $\mathcal{C}_{\mathbf{v}, \alpha}$ (and also the isophote $\mathcal{I}_{\mathbf{v}, \alpha}$ ) is bounded. Hence we have to use the technique formulated for SituATion (ii) and provide rational transformation (11) with $m=20$ (since $p(20)<0$ ). So we obtain a bounded curve $\mathcal{E}^{\prime}$ in the Weierstrass form

$$
y^{2}=-\frac{464128 x^{4}}{27}+\frac{448 x^{3}}{3}+60 x^{2}-x .
$$

Then, we approximate $\mathcal{E}^{\prime}$, see Fig. 5 (c), and using the inverse transformation (13) we obtain an approximation of $\mathcal{E}$. Finally we employ the inverse Weierstrass transformation which yields approximate parametrization of $\mathcal{C}_{\mathbf{v}, \alpha}$ and substituting it to (24) we arrive at an approximate parametric description of the isophote $\mathcal{I}_{\mathbf{v}, \alpha}$, see Fig. $5(\mathrm{~d})$.

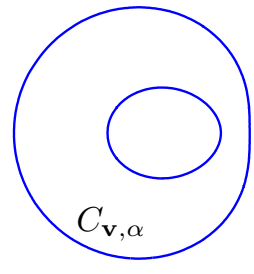

(a)

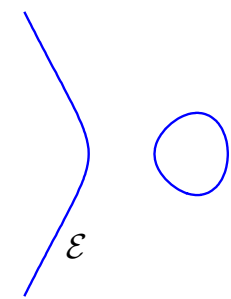

(b)

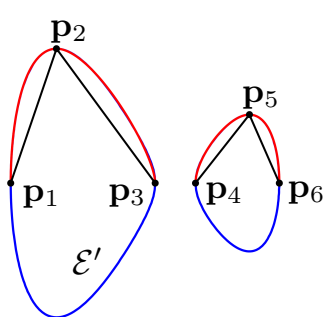

(c)

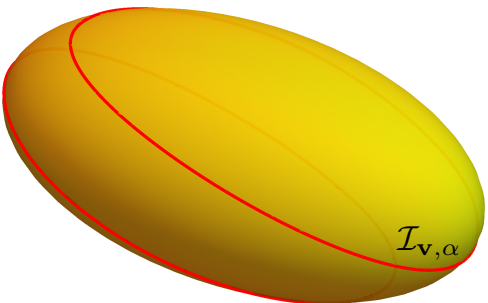

(d)

Figure 5: A curve $\mathcal{C}_{\mathbf{v}, \alpha}$ in the parametric space corresponding to the ispohote $\mathcal{I}_{\mathbf{v}, \alpha}$ (a), its corresponding curve in the Weierstrass form (b), the associated bounded curve in the Weierstrass form with its graph of critical points (c) and an approximation of the isophote $\mathcal{I}_{\mathbf{v}, \alpha}$ on the ellipsoid (d) from Example 4.6.

The second example of approximating the intersection of a ruled surface with a quadric is devoted to computing the intersection curve of two quadric surfaces (QSIC), which is a fundamental problem e.g. in computer graphics, solid modelling etc. There is plenty of work on the classification of a general QSIC, variation of the types of the intersection and the computation of parameterizations, see e.g. Jia et al. (2016) and references therein. For the sake of brevity we recall at least a method from Wang et al. (2002) which is based on a birational mapping between the QSIC and a plane cubic curve, a result well known in the literature of algebraic geometry. In that paper an algorithm for the classification of a general QSIC and the computation of parameterizations of all its irreducible or connected components was developed.

It is beyond the scope of this paper to solve all particular situations. We mainly plan to present another useful application of our unifying approximate approach based on the Weierstrass form of the hyperelliptic curves and show that this technique can also be suitable for approximating some special cases of QSIC. Application of our method is based on the following observation. When the pencil $\Sigma: \lambda_{1} f+\lambda_{2} g=0$ determined by two quadrics $f=0, g=0$ contains a real singular quadric (i.e., a cone or a cylinder being ruled surfaces), the Weierstrass form of QSIC can be easily obtained and approximated by our rationalruled-surface-to-quadric intersection approach. We demonstrate it on the following example.

Example 4.7. Our goal is to compute the intersection of two quadrics, namely of the ellipsoid and onesheeted hyperboloid

$$
f=x^{2}+2 y^{2}+z^{2}-9=0, \quad g=x^{2}-x y-2 x+y^{2}-z^{2}=0 .
$$


In the pencil $\Sigma: \lambda_{1} f+\lambda_{2} g=0$ determined by these two quadrics we can find a singular quadric, in this case the cylinder

$$
h=2 x^{2}-x y-2 x+3 y^{2}-9=0 .
$$

Parameterizing cylinder (29) in the form $\mathbf{x}(s, t)=\mathbf{p}(s)+t(0,0,1)$, where $\mathbf{p}(s)$ is a rational parameterization of the base ellipse $h=0, z=0$

$$
\mathbf{p}(s)=\left(\frac{-22 s^{2}-162 s+351}{23\left(s^{2}-s+6\right)}, \frac{-103 s^{2}+402 s+486}{69\left(s^{2}-s+6\right)}, 0\right)
$$

and intersecting it e.g. with $g=0$ we arrive at the equation

$-4761 s^{4} t^{2}+17275 s^{4}+9522 s^{3} t^{2}+15774 s^{3}-61893 s^{2} t^{2}+336861 s^{2}+57132 s t^{2}-272160 s-171396 t^{2}-38637=0$,

which is quadratic in $t$. Next, computing its discriminant with respect to $t$ we obtain a curve $\mathcal{E}$ in the Weierstrass form

$$
y^{2}=17275 s^{4}+15774 s^{3}+336861 s^{2}-272160 s-38637
$$

and thus we can again apply the designed approach. Since (32) is an unbounded curve, see Fig. 6 (left), in this case, we use transformation (11) for $m=1 / 3$ and obtain a bounded curve $\mathcal{E}^{\prime}$ in the Weierstrass form

$$
y^{2}=-\frac{7381571 s^{4}}{81}-\frac{1073756 s^{3}}{27}+\frac{1092455 s^{2}}{3}+\frac{116422 s}{3}+17275 .
$$

Finally we approximate $\mathcal{E}^{\prime}$, see Fig. 6 (middle), which gives us an approximation of the QSIC, see Fig. 6 (right).
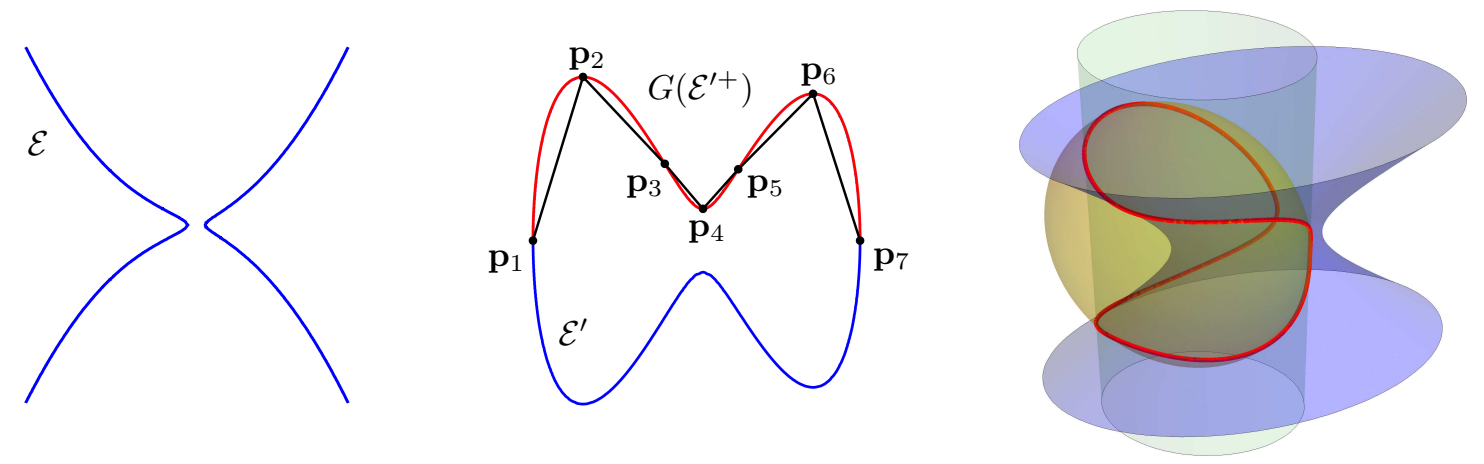

Figure 6: Left: The unbounded Weierstrass curve $\mathcal{E}$. Middle: The graph of critical points $G\left(\mathcal{E}^{\prime+}\right)$ of the bounded Weierstrass curve $\mathcal{E}^{\prime}$. Right: The intersection curve of the ellipsoid and the hyperboloid (and the cylinder) from Example 4.7.

\section{Planar algebraic operations leading to square-root parameterizations}

Current geometric modelling studies and uses many operations defined over algebraic varieties whose output are again algebraic varieties (offsetting, bisectors, operation of conchoid, etc.). Nevertheless, an uncomfortable feature of these constructions is the fact that the output variety may be, in some sense, more complicated than the input varieties - e.g. its algebraic degree is often higher, it consists of more components and mainly it does not have to be rational although the both input objects are rational. In this section we recall some well-known operations/constructions with rational planar curves which do not preserve rationality, in general. We will deal with examples that lead to hyperelliptic curves and especially we focus on operations that can be derived from intersections of ruled surfaces, cf. Section 4.2. 


\subsection{Offsets}

Offsetting is one of the fundamental operations in computer aided design and other practical applications (e.g. numerical-control machining, robot path-planning, tolerance analysis). Due to their high applicability, studying offsets has become a popular research field in the recent past. Many interesting problems related to this topic have been explored, including analysis of their geometric and algebraic properties, determining the number and type of components, constructing rational parameterizations (if they exist) and also approximate techniques for computation and interrogation of non-rational offsets, for more details see e.g. Arrondo et al. (1997, 1999); Maekawa (1999); Sendra and Sendra (2000); Farouki (2008).

Definition 5.1. Let $\mathcal{C}$ be a plane algebraic curve and $\delta \in \mathbb{R}$ a positive constant. Denote by $\mathbf{n}_{\mathbf{p}}$ one of the two unit normal vectors at a regular point $\mathbf{p}$ of $\mathcal{C}$. Then the $\delta$-offset $\mathcal{O}_{\delta} \mathcal{C}$ is the closure of the set

$$
\left\{\mathbf{p} \pm \delta \mathbf{n}_{\mathbf{p}} \mid \mathbf{p} \in \mathcal{C} \backslash \operatorname{Sing}(\mathcal{C})\right\} .
$$

If $\mathcal{C}$ is a rational curve given by the parameterization $\mathbf{x}(t)=\left(x_{1}(t), x_{2}(t)\right)$ then the two branches of the offset yield the parameterization

$$
\mathbf{x}_{\delta}^{ \pm}(t)=\left(x_{1}(t), x_{2}(t)\right) \pm \delta \frac{\left(-x_{2}^{\prime}(t), x_{1}^{\prime}(t)\right)}{\sqrt{x_{1}^{\prime}(t)^{2}+x_{2}^{\prime}(t)^{2}}}
$$

Clearly, the operation of offsetting does not preserve the rationality, in general. In particular starting with a rational curve one obtains in general a square-root parameterization of its offsets. A study of offset rationality led to the class of planar Pythagorean hodograph $(\mathrm{PH})$ curves introduced in Farouki and Sakkalis (1990). These curves are defined as rational curves $\mathbf{x}(t)=\left(x_{1}(t), x_{2}(t)\right)$ fulfilling the distinguishing condition

$$
\mathbf{x}^{\prime}(t) \cdot \mathbf{x}^{\prime}(t)=x_{1}^{\prime}(t)^{2}+x_{2}^{\prime}(t)^{2}=\sigma(t)^{2},
$$

where the parametric speed $\sigma(t)$ is a rational function and ' '. is the standard Euclidean inner product. Since the rationality of the $\delta$-offset curve $\mathbf{x}_{\delta}^{ \pm}(t)$ of a rational curve only depends on the rationality of the associated unit normal field $\mathbf{n}(t)$, planar $\mathrm{PH}$ curves possess rational offsets. Thus, when approximating a given curve by a (piece-wise) PH curve we have guaranteed that the resulting approximate offsets will be rational, see Farouki (2008) for further details about PH curves.

Recalling the results from Arrondo et al. (1997); Vršek and Lávička (2013) dealing with the reducibility of offsets, and using the terminology from Vršek and Lávička (2014) we may classify the rational curves:

Definition 5.2. A rational curve $\mathcal{C}$ is said to be proper $\mathrm{PH}$, non-proper $\mathrm{PH}$, or non- $\mathrm{PH}$ if every its parameterization is $\mathrm{PH}$, there exist both $\mathrm{PH}$ and non-PH parameterizations, or there exists no PH parameterization, respectively.

If the curve is non-PH then its offsets are non-rational, however they admit square-root parameterizations and thus they are hyperelliptic by Lemma 2.3. If we write

$$
\sqrt{\mathbf{x}^{\prime}(t) \cdot \mathbf{x}^{\prime}(t)}=\frac{q(t)}{r(t)} \sqrt{p(t)},
$$

where $p(t)$ is a square-free polynomial, then we arrive at:

Lemma 5.3. Any offset of a non-PH curve is a hyperelliptic curve with Weierstrass form (1), where the square-free polynomial $p$ is obtained from (37).

Applying the general method described in Section 3 for the approximation of curves in the Weierstrass form suggests an alternative way (compared to standard interpolation techniques based on PH curves) how to construct approximate rational offsets. 
Example 5.4. Consider (a bounded part of) the cubic

$$
\mathbf{x}(t)=\left(t^{3}, t\right), \quad t \in[-2,2],
$$

then the 2-offset has the square-root parameterization (35) of the form

$$
\mathbf{x}_{2}^{ \pm}(t)=\left(t^{3} \pm \frac{2}{\sqrt{9 t^{4}+1}}, t \mp \frac{6 t^{2}}{\sqrt{9 t^{4}+1}}\right), \quad t \in[-2,2] .
$$

Hence our goal is to approximate the curve $\mathcal{E}$ in the Weierstrass form

$$
y^{2}-9 x^{4}-1=0, \quad x \in[-2,2] .
$$

We construct the graph of critical points $G\left(\mathcal{E}^{+}\right)$, see Fig. 7 (left). In particular $G\left(\mathcal{E}^{+}\right)$contains only two edges which will be replaced by the Ferguson cubics. Finally (3) yields the piecewise rational approximation of the offset curve (39), see Fig. 7 (right).
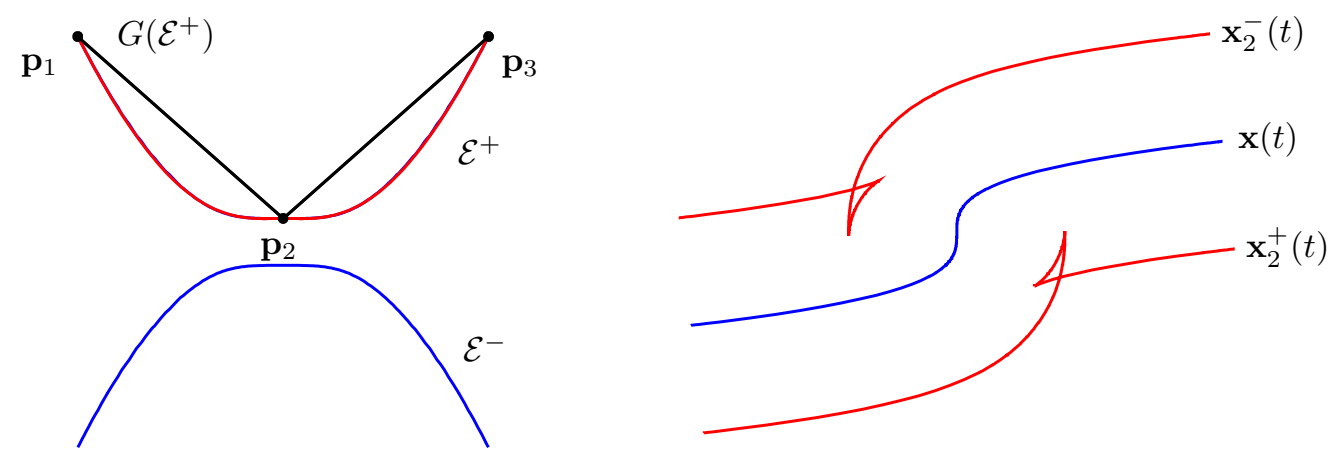

Figure 7: The graph of critical points $G\left(\mathcal{E}^{+}\right)$of the curve $\mathcal{E}$ (left) and the cubic curve (blue) with the computed rational approximation of its two-sided offset in red (right) from Example 5.4.

Remark 5.5. As the polynomial $p(t)$, cf. (37), does not depend on $\delta$ we obtained approximations of all the offsets at the same time. Hence approximating the hyperelliptic curve $y^{2}-9 x^{4}-1=0$ by a piecewise polynomial curve is a problem equivalent to the approximation of the given cubic $\left(t^{3}, t\right)$ by a piecewise $\mathrm{PH}$ curve.

To conclude this subsection we will show that the $\delta$-offset of any given curve is nothing but the intersection of a special ruled surface $\Gamma_{\mathcal{C}} \subset \mathbb{R}^{3}$ defined as the closure of

$$
\left\{(\mathbf{p}, 0) \pm t\left(n_{\mathbf{p}}, 1\right) \mid \mathbf{p} \in \mathcal{C} \backslash \operatorname{Sing}(\mathcal{C}) \text { and } t \in \mathbb{R}\right\}
$$

with the plane $z=\delta$, see Fig. 8 (left). These surfaces, called also isotropic surfaces, were studied in Peternell and Pottmann (1998); Pottmann and Peternell (1998); Krasauskas and Mäurer (2000); Kosinka and Lávička (2010) in connection with PH/MPH curves. They are non-rational in general and their properties are, in fact, closely related to the existence of $\mathrm{PH}$ parameterizations of the base curve, as the following theorem shows.

Theorem 5.6. Let $\mathcal{C}$ be a rational plane curve. Then $\Gamma_{\mathcal{C}}$

(i) consists of two rational ruled surfaces $\Gamma_{\mathcal{C}}^{+} \cup \Gamma_{\mathcal{C}}^{-}$symmetric w.r.t the plane $z=0$ iff $\mathcal{C}$ is a proper $P H$ curve;

(ii) is an irreducible rational ruled surface iff $\mathcal{C}$ is a non-proper $\mathrm{PH}$ curve; 

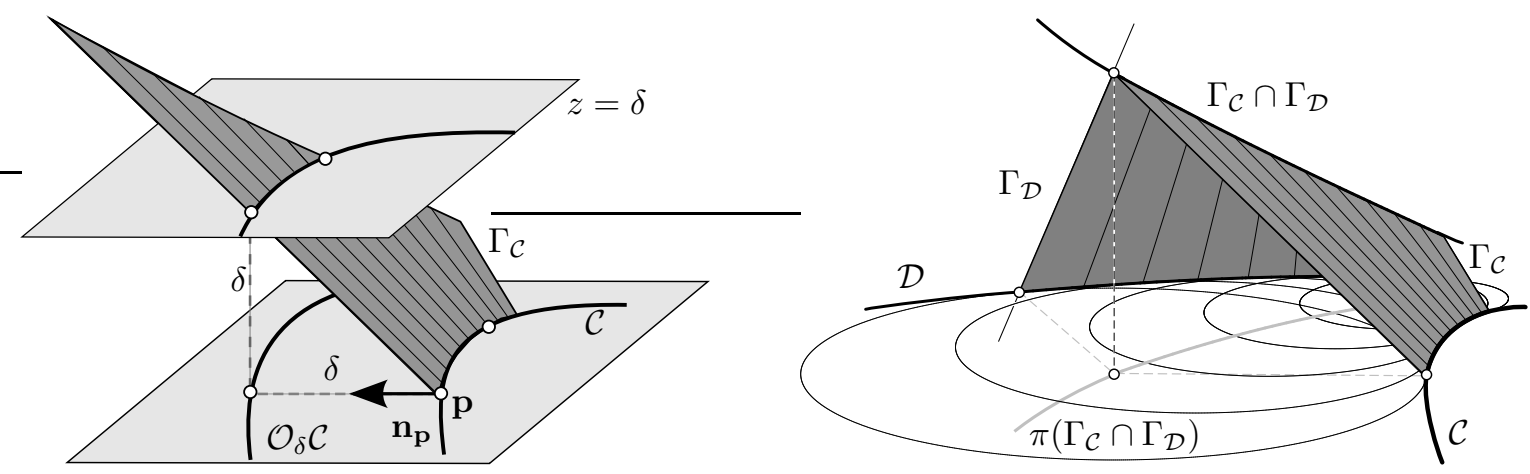

Figure 8: Left: The intersection of the isotropic surface $\Gamma_{\mathcal{C}}$ with the plane $z=\delta$ yielding the $\delta$-offset of $\mathcal{C}$. Right: The bisectors of two curves $\mathcal{C}$ and $\mathcal{D}$ obtained using their isotropic surfaces.

(iii) is an irreducible non-rational ruled surface with hyperelliptic plane sections iff $\mathcal{C}$ is a non-PH curve.

Proof. A PH parameterization $\mathbf{c}(t)$ of $\mathcal{C}$ provides a rational unit normal field of the curve $\mathbf{n}(t)=\mathbf{c}^{\prime}(t)^{\perp} / \sigma(t)$, where $\sigma^{2}=\mathbf{c}^{\prime} \cdot \mathbf{c}^{\prime}$ and thus leads to a rational parameterization of $\Gamma_{\mathcal{C}}$ via $(\mathbf{c}(t), 0)+s(\mathbf{n}(t), 1)$. Hence, this is a rational ruled surface. Moreover if $\mathbf{c}(t)$ is birational then $(\mathbf{c}(t), 0) \pm s(\mathbf{n}(t), 1)$ parameterizes two distinct surfaces and it is easy to see that these are exactly $\Gamma_{\mathcal{C}}^{+}$and $\Gamma_{\mathcal{C}}^{-}$. A non-proper PH curve does not possess a birational $\mathrm{PH}$ parametrization but there exists a $\mathrm{PH}$ parametrization which traces this curve twice, i.e., for a generic point $\mathbf{p}$ there exist two values $t_{1}, t_{2}$ such that $\mathbf{c}\left(t_{1}\right)=\mathbf{c}\left(t_{2}\right)=\mathbf{p}$ and $\mathbf{n}\left(t_{1}\right)=-\mathbf{n}\left(t_{2}\right)$, see Arrondo et al. (1997). Hence the single parameterization parameterizes the whole $\Gamma_{\mathcal{C}}$ and it must be irreducible. The curve $\mathcal{C}$ is then a double curve on the surface.

Conversely let $\mathbf{p}(t)+s \mathbf{q}(t)$ be a parameterization of $\Gamma_{\mathcal{C}}$ (or one of its components). Then the intersection with the plane $z=0$ is a rational curve parameterized by

$$
\left(p_{1}(t)-\frac{p_{3}(t)}{q_{3}(t)} q_{1}(t), p_{2}(t)-\frac{p_{3}(t)}{q_{3}(t)} q_{2}(t), 0\right) .
$$

This is a PH parameterization because it possesses a unit normal field $\left(q_{1}(t) / q_{3}(t), q_{2}(t) / q_{3}(t), 0\right)$. Again if $\Gamma_{\mathcal{C}}$ is irreducible then $\mathcal{C}$ is a double curve and its generic point lies on two rulings corresponding to two values of $t$. Hence the resulting PH parameterization cannot be birational. This proves (i) and (ii).

We already saw that a non-PH curve produces a non-rational ruled surface $\Gamma_{\mathcal{C}}$. Consider a variety

$$
\Phi:=\left\{(\mathbf{p}, \mathbf{q}) \in \mathcal{C} \times \Gamma_{\mathcal{C}} \mid \mathbf{q} \text { lies on a ruling passing through } \mathbf{p}\right\}
$$

and the projection $\mu: \Phi \rightarrow \mathcal{C}$. Since a generic point $\mathbf{p} \in \mathcal{C}$ is contained in exactly two lines and a line intersects a plane in one point, the restriction of $\mu$ to the intersection of $\Phi$ with a generic plane is a double cover of a rational curve $\mathcal{C}$. Hence the plane section of $\Gamma_{\mathcal{C}}$ is a hyperelliptic curve. This concludes the proof of (iii).

Example 5.7. The following description of isotropic surfaces of curves of degree 1 and 2 is an immediate consequence of Theorem 5.6.

1. If $\mathcal{L}$ is a line then $\Gamma_{\mathcal{L}}$ consists of two planes.

2. If $\mathcal{S}^{1}$ is a circle then $\Gamma_{\mathcal{S}^{1}}$ consists of two circular cones.

3. If $\mathcal{P}$ is a parabola then $\Gamma_{\mathcal{P}}$ is a rational ruled surface of degree 6 .

4. If $\mathcal{Q}$ is any other regular conic section then $\Gamma_{\mathcal{Q}}$ is a ruled surface with sectional genus 1 .

In addition, let $\pi: \mathbb{R}^{3} \rightarrow \mathbb{R}^{2}$ be the natural projection forgetting the third coordinate. Then we immediately obtain 
Lemma 5.8. The $\delta$-offset of the curve $\mathcal{C}$ is the image of the intersection of $\Gamma_{\mathcal{C}}$ with the plane $z=\delta$ under the projection $\pi$.

Proof. We kindly refer the readers to Pottmann and Peternell (1998).

Remark 5.9. Obviously, Theorem 5.6 and Lemma 5.8 confirm the hyperelipticity of the offsets of rational curves, discussed before.

Remark 5.10. Let us recall the paper by Peternell et al. (2015) in which a close relation of conchoids to offsets is thoroughly investigated. Consider an algebraic curve $\mathcal{C}$, a point $\mathbf{o}$ and a positive constant $\delta \in \mathbb{R}$. If $\leftrightarrow \mathbf{o p}$ denotes the line joining $\mathbf{o}$ and $\mathbf{p}$ then the $\delta$-conchoid $\mathcal{C}_{\delta} \mathcal{C}$ is the closure of the set

$$
\{\mathbf{q} \in \leftrightarrow \mathbf{o p} \mid \mathbf{p} \in \mathcal{C} \text { and }\|\mathbf{q}-\mathbf{p}\|=\delta\} .
$$

It is shown in Peternell et al. (2015) that a family of offsets corresponds birationally to a family of conchoids. This confirms the hyperelliptic feature of conchoids of rational curves (as in the offset case) and justifies the application of the same designed approximation approach.

Example 5.11. Consider the circle

$$
\mathbf{x}(t)=\left(\frac{1-t^{2}}{t^{2}+1}, \frac{2 t}{t^{2}+1}\right), \quad t \in \mathbb{R},
$$

the point $\mathbf{o}=(-3 / 2,1)$ and $\delta=3 / 2$. Then the corresponding conchoid has the parameterization

$$
\mathbf{c}^{ \pm}(t)=\left(\frac{1-t^{2}}{t^{2}+1} \pm \frac{3\left(t^{2}+5\right)}{2 \sqrt{5 t^{4}-16 t^{3}+34 t^{2}-16 t+29}}, \frac{2 t}{t^{2}+1} \mp \frac{3(t-1)^{2}}{\sqrt{5 t^{4}-16 t^{3}+34 t^{2}-16 t+29}}\right) .
$$

According to the presented approach we approximate the curve

$$
y^{2}=5 x^{4}-16 x^{3}+34 x^{2}-16 x+29, \quad x \in \mathbb{R} .
$$

The curve in the Weierstrass form $\mathcal{E}$ given by (47) is always unbounded because $p(t) \geq 0$ for all $t \in \mathbb{R}$. So we can use the same procedure as in Example 5.16. Finally, we obtain an approximation of the whole curve $\mathcal{E}$, see Fig. 9.

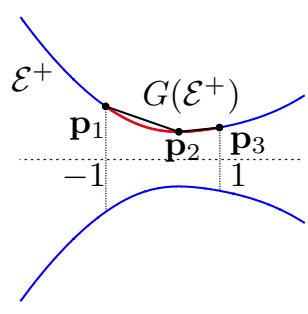

(a)

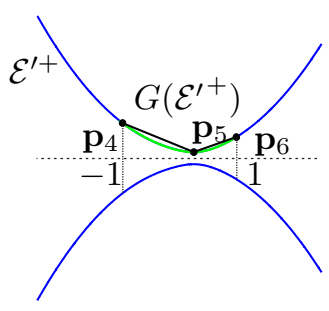

(b)

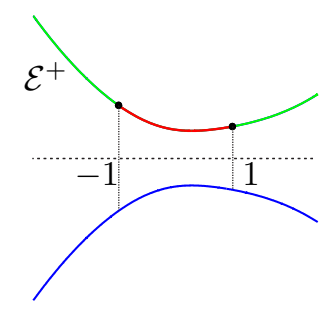

(c)

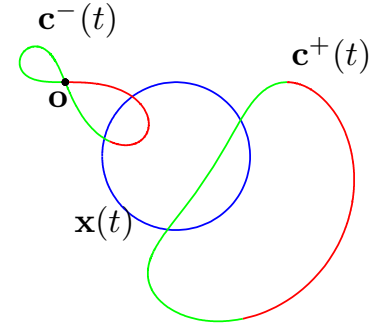

(d)

Figure 9: The curves $\mathcal{E}$ and $\mathcal{E}^{\prime}$ given by the Weierstrass form with their rational approximations on $[-1,1]$ in red and green, respectively (a), (b). The approximation of the whole $\mathcal{E}^{+}$(c) and the approximation of the whole conchoid (d) from Example 5.11. 


\subsection{Bisectors}

Bisectors are closely related to skeletons and curved Voronoi diagrams, structures thoroughly studied in geometric modelling or computational geometry, and used in many technical applications as $\mathrm{NC}$ pocket machining, finite element mesh generation, collision-avoidance motion planning etc., see e.g. Farin et al. (2002). To construct a skeleton or a curved Voronoi diagram, first we need to determine an arrangement of bisector curves, i.e., for each pair of input planar curves (determining the boundary) we have to find a locus of all points with equal orthogonal distance to both given curves. This property also justifies alternative names equidistant curves or medial axes for the bisectors.

Definition 5.12. Let $\mathcal{C}$ and $\mathcal{D}$ be two planar curves. Denote $N_{\mathbf{p}} \mathcal{C}$ and $N_{\mathbf{q}} \mathcal{D}$ the normal lines at regular points $\mathbf{p} \in \mathcal{C}$ and $\mathbf{q} \in \mathcal{D}$, respectively. Then the bisector $\mathcal{B}(\mathcal{C}, \mathcal{D})$ of curves is defined as the closure of the set

$$
\left\{\mathbf{x} \in \mathbb{R}^{2} \mid \exists \mathbf{p} \in \mathcal{C}, \exists \mathbf{q} \in \mathcal{D}: \mathbf{x}=N_{\mathbf{p}} \mathcal{C} \cap N_{\mathbf{q}} \mathcal{D} \text { and }(\mathbf{x}-\mathbf{p})^{2}=(\mathbf{x}-\mathbf{q})^{2}\right\} .
$$

For further details about bisectors and computing with them (including some approximation methods) we refer e.g. to Farouki and Johnstone (1994); Elber and Kim (1998); Oliveira and De Figueiredo (2003); Adamou et al. (2014). Recently, a general theoretical study from the point of view of the algebraic geometry was presented in Fioravanti and Sendra (2016). In that paper the bisectors were obtained by projection from the so-called incidence variety and some algebro-geometric analysis was provided.

Following the approach from the previous parts devoted mainly to ruled surfaces and their intersections, the bisectors of two curves can be also obtained this way, namely using their isotropic surfaces, see Fig. 8 (right).

Lemma 5.13. The bisector of the curves $\mathcal{C}$ and $\mathcal{D}$ is the closure of the set $\pi\left(\Gamma_{\mathcal{C}} \cap \Gamma_{\mathcal{D}}\right)$.

Proof. We kindly refer the readers to Pottmann and Peternell (1998).

Since $\left(\mathbf{p}, p_{z}\right) \in \Gamma_{\mathcal{C}} \cap \Gamma_{\mathcal{D}}$ implies $\left(\mathbf{p},-p_{z}\right) \in \Gamma_{\mathcal{C}} \cap \Gamma_{\mathcal{D}}$ the projection is not birational. However if at least one of the curves, let us say $\mathcal{C}$, is a proper $\mathrm{PH}$ curve then $\Gamma_{\mathcal{C}}=\Gamma_{\mathcal{C}}^{+} \cup \Gamma_{\mathcal{C}}^{-}$and we may write the bisector as $\pi\left(\Gamma_{\mathcal{C}}^{+} \cap \Gamma_{\mathcal{D}}\right)$.

The above observation together with the results from Example 5.7 enable us to identify classes of curves yielding rational or hyperelliptic bisectors.

Corollary 5.14. The bisector of a line and a rational curve is hyperelliptic. Moreover if the curve is PH then the bisector is rational.

Proof. It follows immediately from Lemma 5.8, Example 5.7 and the fact that the plane section of a rational ruled surface is a rational curve. In addition, let us recall that the subresult concerning the rationality appeared already in Peternell (2000).

Moreover we can formulate an analogous result also for bisectors with circles.

Corollary 5.15. The bisector of a circle and a rational curve is hyperelliptic. Moreover if the curve is PH then the bisector is rational.

Proof. The surface $\Gamma_{\mathcal{S}^{1}}$ consists of two circular cones. Hence the bisector is a projection of $\Gamma_{\mathcal{S}^{1}}^{+} \cap \Gamma_{\mathcal{C}}$. Let $\mathcal{L}$ be a ruling on the isotropic surface $\Gamma_{\mathcal{C}}$ then it intersects $\Gamma_{\mathcal{S}^{1}}^{+}$in two points by the Bézout theorem. However there always exists a ruling $\mathcal{M}$ on $\Gamma_{\mathcal{S}^{1}}^{+}$parallel to $\mathcal{L}$ and thus one of the intersection points is in the plane at infinity. It turns out that whenever $\mathcal{C}$ is not a line, $\Gamma_{\mathcal{S}^{1}}^{+} \cap \Gamma_{\mathcal{C}}$ contains a conic section at infinity. And thus the affine part of $\Gamma_{\mathcal{S}^{1}}^{+} \cap \Gamma_{\mathcal{C}}$ intersects the ruling in one point which means that it is a section on the ruled surface $\Gamma_{\mathcal{C}}$. Hence using Theorem 5.6 we conclude that the bisector of a circle and a proper PH curve, a non-proper $\mathrm{PH}$ curve, and a rational non-PH curve consists of two rational curves, one rational curve, or one hyperelliptic curve, respectively. 
Example 5.16. Whereas the isotropic surfaces are especially useful for theoretical reasoning, the approach via parameter spaces, see e.g. Elber and Kim (1998), is more suitable for explicit computations.

Consider the ellipse and the circle

$$
\mathbf{x}_{1}(u)=\left(\frac{4 u}{u^{2}+1}, \frac{3\left(u^{2}-1\right)}{2\left(u^{2}+1\right)}\right), \quad \mathbf{x}_{2}(v)=\left(\frac{2 v}{v^{2}+1}, \frac{v^{2}-1}{v^{2}+1}\right) .
$$

Then the bisector $\mathbf{b}(t)$ is determined by the following conditions:

$$
\begin{aligned}
\mathbf{b}_{1}(u, v)=\mathbf{x}_{1}(u)+\alpha(u, v) \mathbf{n}_{1}(u) & =\mathbf{x}_{2}(v)+\beta(u, v) \mathbf{n}_{2}(v)=\mathbf{b}_{2}(u, v), \\
\left\|\alpha(u, v) \mathbf{n}_{1}(u)\right\|^{2} & =\left\|\beta(u, v) \mathbf{n}_{2}(v)\right\|^{2},
\end{aligned}
$$

where $\alpha, \beta \in \mathbb{R}(u, v)$ and $\mathbf{n}_{1}(u)=\left(x_{12}^{\prime},-x_{11}^{\prime}\right)$ and $\mathbf{n}_{2}(v)=\left(x_{22}^{\prime},-x_{21}^{\prime}\right)$ are normal vectors of $\mathbf{x}_{1}(u)$ and $\mathbf{x}_{2}(v)$, respectively. This yields a condition for the parameters $u$ and $v$ in the form

$$
\left(\left(\mathbf{x}_{2}(v)-\mathbf{x}_{1}(u)\right) \cdot \mathbf{x}_{2}^{\prime}(v)\right)^{2}\left\|\mathbf{n}_{1}(u)\right\|^{2}=\left(\left(\mathbf{x}_{2}(v)-\mathbf{x}_{1}(u)\right) \cdot \mathbf{x}_{1}^{\prime}(v)\right)^{2}\left\|\mathbf{n}_{2}(v)\right\|^{2} .
$$

In this particular case we obtain

$$
20 u^{6} v-29 u^{5} v^{2}+85 u^{5}-92 u^{4} v-30 u^{3} v^{2}+30 u^{3}+92 u^{2} v-85 u v^{2}+29 u-20 v=0,
$$

which is quadratic in $v$ and thus we can use again the presented method for computing its approximation. Namely, the discriminant of (52), after the factorization, gives the curve $\mathcal{E}$ in the Weierstrass form

$$
y^{2}=4 u^{4}+u^{2}+4 .
$$

The curve in Weierstrass form $\mathcal{E}$ given by (53) is unbounded for $x \in \mathbb{R}$ - in particular, it represents Situation (iii). Thus, we compute first its approximation only in some closed interval, e.g. for $x \in[-1,1]$, see Fig. 10 (a). And subsequently, we perform a rational transformation (11) for $m=0$ which yields the curve $\mathcal{E}^{\prime}$, where the part $x \in[-1,1]$ corresponds to the interval $(-\infty,-1) \cup(1,+\infty)$ on $\mathcal{E}$. We approximate $\mathcal{E}^{\prime}$ for $x \in[-1,1]$, see Fig. 10 (b), and employing (13) we return back on $\mathcal{E}$. Now, we have obtained an approximation of the whole curve $\mathcal{E}$, see Fig. 10 (c), consisting of four parts. Using (3) we arrive at the sought approximation of the bisector, see Fig. 10 (d).

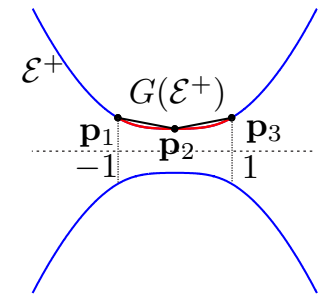

(a)

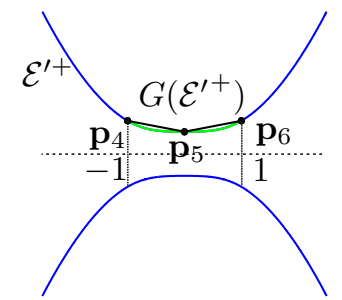

(b)

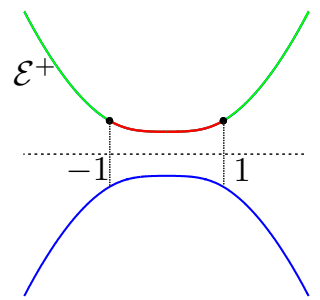

(c)

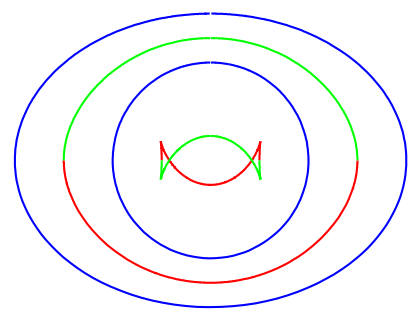

(d)

Figure 10: The curves $\mathcal{E}$ and $\mathcal{E}^{\prime}$ given by Weirestrass form with their rational approximations on [-1,1] in red and green, respectively (a),(b). The approximation of the whole $\mathcal{E}^{+}$(c) and the approximation of the whole bisector of the ellipse and the circle (d) from Example 5.16.

\section{Conclusion}

We focused on several situations originated in geometric modelling when non-rational parameterizations of planar or space curves as results of certain geometric constructions are gained. Especially we studied the 
cases in which one can identify a rational mapping that is a double cover of a rational curve, i.e., when the square-root parameterizations typically arise and thus suitable rational approximate techniques are needed. As a unifying scheme we revealed the approach based on studying intersections of ruled surfaces which can be used also for explanations of various planar algebro-geometric operations.

We designed a simple algorithm for computing an approximate rational parametrization of hyperelliptic curves using topological graphs of their Weierstrass form. Predictable shapes reflecting a number of real roots of a univariate polynomial and a possibility to approximate easily the components of the Weierstrass curve separately play a main role in our approximation technique. The functionality of the formulated algorithm was demonstrated on several examples.

\section{Acknowledgments}

The authors were supported by the project LO1506 of the Czech Ministry of Education, Youth and Sports. We thank to all referees for their valuable comments, which helped us to improve the paper.

\section{References}

Adamou, I., Fioravanti, M., Gonzalez-Vega, L., Mourrain, B., 2014. Bisectors and Voronoï Diagram of a Family of Parallel Half-Lines. Springer International Publishing, Cham, pp. 241-279.

Alcázar, J., Sendra, J. R., June 2005. Computation of the topology of real algebraic space curves. Journal of Symbolic Computation 39, 719-744.

URL http://dx.doi.org/10.1016/j.jsc.2005.01.006

Arrondo, E., Sendra, J., Sendra, J. R., 1997. Parametric generalized offsets to hypersurfaces. Journal of Symbolic Computation $23,267-285$

Arrondo, E., Sendra, J., Sendra, J. R., 1999. Genus formula for generalized offset curves. Journal of Pure and Applied Algebra 136, 199-209.

Bizzarri, M., Lávička, M., Vršek, J., 2015. Canal surfaces with rational contour curves and blends bypassing the obstacles. Computer-Aided Design 64, 55 - 67.

Bizzarri, M., Lávička, M., 2013a. Parameterizing rational offset canal surfaces via rational contour curves. Computer-Aided Design 45 (2), 342-350.

Bizzarri, M., Lávička, M., 2013b. A symbolic-numerical approach to approximate parameterizations of space curves using graphs of critical points. Journal of Computational and Applied Mathematics 242, $107-124$.

Bizzarri, M., Lávička, M., Kosinka, J. K., 2016. Medial axis transforms yielding rational envelopes. Computer Aided Geometric Design 46, $92-102$.

Cheng, J., Lazard, S., Peñaranda, L., Pouget, M., Rouillier, F., Tsigaridas, E., 2009. On the topology of planar algebraic curves. In: Proceedings of the 25th annual symposium on Computational geometry. SCG '09. ACM, New York, NY, USA, pp. $361-370$.

Cheng, J.-S., Jin, K., Lazard, D., 2013. Certified rational parametric approximation of real algebraic space curves with local generic position method. Journal of Symbolic Computation 58, 18-40.

Elber, G., Kim, M.-S., 1998. Bisector curves of planar rational curves. Computer-Aided Design 30 (14), 1089 - 1096.

Farin, G., Hoschek, J., Kim, M.-S. (Eds.), 2002. Handbook of Computer Aided Geometric Design. Elsevier.

Farouki, R., 2008. Pythagorean-Hodograph Curves: Algebra and Geometry Inseparable. Springer.

Farouki, R., Sakkalis, T., 1990. Pythagorean hodographs. IBM Journal of Research and Development 34 (5), 736-752.

Farouki, R. T., Johnstone, J. K., 1994. The bisector of a point and a plane parametric curve. Comput. Aided Geom. Des. $11(2), 117-151$.

Fioravanti, M., Sendra, J. R., 2016. Algebro-geometric analysis of bisectors of two algebraic plane curves. Computer Aided Geometric Design 47, 189 - 203, sI: New Developments Geometry.

Gamelin, T. W., Greene, R. E., 1999. Introduction to topology. Dover Publications.

Hartshorne, R., 1977. Algebriac Geometry. Springer-Verlag.

Hong, H., 1996. An efficient method for analyzing the topology of plane real algebraic curves. In: Selected papers presented at the international IMACS symposium on Symbolic computation, new trends and developments. Elsevier Science Publishers B. V., Amsterdam, The Netherlands, The Netherlands, pp. 571-582.

Hoschek, J., Lasser, D., 1993. Fundamentals of Computer Aided Geometric Design. AK Peters.

Jia, X., Wang, W., Choi, Y.-K., Mourrain, B., Tu, C., 2016. Continuous detection of the variations of the intersection curve of two moving quadrics in 3-dimensional projective space. Journal of Symbolic Computation 73 (C), $221-243$.

Jüttler, B., Chalmovianský, P., January 2007. A predictor-corrector-type technique for the approximate parameterization of intersection curves. Applicable Algebra in Engineering, Communication and Computing 18, $151-168$.

Kosinka, J., Lávička, M., 2010. On rational Minkowski Pythagorean hodograph curves. Computer Aided Geometric Design 27 (7), 514-524.

Krasauskas, R., Mäurer, C., 2000. Studying cyclides with Laguerre geometry. Computer Aided Geometric Design 17 (2), $101-126$. 
Maekawa, T., 1999. An overview of offset curves and surfaces. Computer-Aided Design 31, $165-173$.

Oliveira, J. B., De Figueiredo, L. H., 2003. Robust approximation of offsets, bisectors, and medial axes of plane curves. Reliable Computing 9 (2), 161-175.

Pérez-Díaz, S., Sendra, J. R., Rueda, S. L., Sendra, J., February 2010. Approximate parametrization of plane algebraic curves by linear systems of curves. Computer Aided Geometric Design 27, 212-231.

Peternell, M., 2000. Geometric properties of bisector surfaces. Graphical Models 62 (3), $202-236$.

Peternell, M., Gotthart, L., Sendra, J., Sendra, J. R., 2015. Offsets, conchoids and pedal surfaces. Journal of Geometry 106 (2), $321-339$

Peternell, M., Pottmann, H., 1998. A Laguerre geometric approach to rational offsets. Computer Aided Geometric Design 15, $223-249$.

Piegl, L., Tiller, W., 1997. The NURBS book, 2nd Edition. Monographs in Visual Communication. Springer-Verlag New York, Inc.

Pottmann, H., Peternell, M., 1998. Applications of Laguerre geometry in CAGD. Computer Aided Geometric Design 15, $165-186$.

Pottmann, H., Wallner, J., 2001. Computational Line Geometry. Springer.

Rueda, S. L., Sendra, J., Sendra, J. R., 2013. An algorithm to parametrize approximately space curves. Journal of Symbolic Computation 56, $80-106$.

Sederberg, T. W., Zhao, J., Zundel, A. K., 1989. Approximate Parametrization of Algebraic Curves. Springer Berlin Heidelberg, Berlin, Heidelberg, pp. 33-54.

Sendra, J. R., Sendra, J., 2000. Algebraic analysis of offsets to hypersurfaces. Mathematische Zeitschrift 237, 697-719.

van Hoeij, M., 1995. An algorithm for computing the Weierstrass normal form. In: ISSAC '95 Proceedings of the 1995 international symposium on Symbolic and algebraic computation. ISSAC '95. ACM, New York, NY, USA, pp. 90-95.

van Hoeij, M., 2002. An algorithm for computing the Weierstrass normal form of hyperelliptic curves. arXiv:math/0203130.

Vršek, J., Lávička, M., 2014. Surfaces with Pythagorean normals along rational curves. Computer Aided Geometric Design 31 (7-8), 451 - 463, recent Trends in Theoretical and Applied Geometry.

Vršek, J., 2016. Contour curves and isophotes on rational ruled surfaces. arXiv:1609.07900 [math.AG].

Vršek, J., Lávička, M., 2013. Reducibility of offsets to algebraic curves. Computer Aided Geometric Design 30 (1), $140-147$.

Wang, W., Joe, B., Goldman, R., 2002. Computing quadric surface intersections based on an analysis of plane cubic curves. Graphical Models 64 (6), 335-367. 